\title{
Changes in diaphragm contractility in cigarette smoking-exposed and smoking cessation rats are associated with alterations in mitochondrial morphology and homeostasis
}

\section{Yijie Zhang}

Capital Medical University

Haiyan Sheng

Capital Medical University

Xiaoqian Shi

Capital Medical University

Yuhan $\mathrm{Hu}$

Capital Medical University

Baosen Pang

Capital Medical University

Yingmin Ma

Capital Medical University

Jiawei Jin ( $\sigma_{\text {jiaweijin@ccmu.edu.cn ) }}$

Capital Medical University

\section{Research Article}

Keywords: Cigarette smoking, mitochondrion, diaphragm, autophagy, fusion, fission

Posted Date: January 25th, 2022

DOI: https://doi.org/10.21203/rs.3.rs-1234866/v1

License: (c) (i) This work is licensed under a Creative Commons Attribution 4.0 International License. Read Full License 


\section{Abstract}

Background: Cigarette smoking (CS) is one of the greatest risk factors for the pathogenesis and progression of chronic obstructive pulmonary disease (COPD). Although CS cessation is beneficial in preventing COPD progression, the effects of cessation on the diaphragm are unknown, as are the CSinduced mitochondrial changes in the diaphragm during COPD and CS-cessation. In this study, we examined the alterations in mitochondrial morphology and homeostasis, as well as changes in diaphragm contractility during CS exposure and after cessation.

Methods: Rats were randomly divided into control, CS-exposure, and CS-cessation groups, including 3month CS (S3), 6-month CS (S6), 6-month CS followed by 3 months cessation (S6N3), and age-matched control groups. The histological and functional changes in the lungs were examined to evaluate the CSinduced COPD model. The alterations in the diaphragm were further investigated, including contractile properties, the ultrastructure, and the expression of markers of mitochondrial homeostasis.

Results: CS exposure caused histological disruption and functional depression in the lungs, and CS cessation failed to result in a significant recovery. CS induced a significant decline in diaphragmatic muscle contractility, accompanied with sever contractile dysfunction in extensor digitorum longus muscles, which was recovered after 3-month CS cessation. CS exposure in parallel disrupted the mitochondrial morphology in diaphragmatic muscle, including decreases in volume density and number density in the S6 group, which was significantly alleviated in the S6N3 group. The mitochondrial quality control was likely depressed in the S6 group, as indicated by the downregulation of Pink1 and Mfn1, markers for mitophagy and mitochondrial fusion/fission. Interestingly, the Mfn1 protein level was recovered after smoking cessation in the S6N3 group.

Conclusions: Smoking cessation eased CS-induced diaphragmatic dysfunction and mitochondrial deregulation, but not the adverse changes in pulmonary structure. These diaphragmatic muscle changes are likely associated with deregulated mitochondrial homeostasis, including mitophagy and mitochondrial fusion/fission.

\section{Background}

Chronic obstructive pulmonary disease (COPD) is characterized by persistent respiratory symptoms and airflow limitations, which are due to airway and/or alveolar abnormalities [1]. Muscle dysfunctions in both respiratory (in particular the diaphragm) and skeletal muscles, as extrapulmonary comorbidities, contribute to exercise intolerance and poor health status in patients with COPD [1]. Respiratory muscle dysfunction increases with the exacerbation of COPD, which is associated with a marked increase in hospital admissions [2]. Intriguingly, the diaphragm undergoes a positive adaptation (training-like effect) to protect against muscle dysfunction in the early stage of COPD [3-6]. In addition, diaphragmatic muscle shows more resistance to atrophy compared to skeletal muscle in patients with COPD, suggesting a diaphragm-specific pathophysiological mechanism in muscle 
dysfunction [7]. However, a recent study showed maladaptive changes, including impaired cytoplasm integrity with enhanced proteolysis, in early COPD involved with cigarette smoking (CS) [8]. This contradiction is likely due to the CS-induced adverse effects on respiratory muscle disrupting this adaptive transition.

CS is one of the greatest risk factors for the pathogenesis and progression of COPD [9-11], and the CSinduced deleterious effects on extrapulmonary muscles, other than the respiratory system, are significantly involved in the pathogenesis of COPD [11-13]. Both clinical studies on smokers and CSexposed animal models have shown impaired skeletal muscle function before the development of respiratory symptoms, suggesting a direct effect of CS on muscles [11-13]. Recent studies have shown that CS-induced skeletal muscle deregulation, including mitochondrial dysfunction and atrophy, was significantly recovered by CS cessation in a mouse model [14]. Although CS exposure also had deleterious effects on the diaphragm [11-13], few studies have investigated whether smoking cessation could recover the diaphragm dysfunction as the respiratory muscle in CS-induced COPD.

CS-induced oxidative stress is an important mechanism of muscle dysfunction in COPD $[11,15]$. The oxidative stress-induced impairments of mitochondrial structural and functional integrity have been highlighted in the pathogenesis of muscle dysfunction in COPD [16-19]. Mitochondria in locomotor muscles from patients with COPD are characterized by decreased oxidation capacity and increased reactive oxygen species (ROS), while CS cessation obviously alleviates mitochondrial dysfunction $[11,19]$. Our previous transcriptome profiling study showed that metabolic gene expression was deregulated along with mitochondrial morphological changes in the diaphragm muscle from CSexposed rats [6]. Of note, the balance between fusion and fission plays a critical role in maintaining mitochondrial morphology, quantity, and function, and its disruption activates mitophagy to identify and eliminate damaged mitochondria via autophagy [19]. Mitophagy plays a crucial role in maintaining cell homeostasis and in the pathogenesis of numerous chronic lung diseases [18].

Previous studies have indicated that CS exposure disrupts mitophagy and the accumulation of damaged mitochondria in a deteriorative state [20-25]. The imbalance of mitochondrial fusion/fission caused by CS exposure increases the susceptibility of the lung to cellular stress and senescence, which contributes to age-related COPD pathogenesis [25]. However, these previous studies focused on the mitochondrial changes in pulmonary epithelial cells, bronchial smooth muscle cells, and skeletal muscle of the limbs in the pathogenesis of COPD [20-24], and the mitochondrial autophagy and homeostasis in the diaphragm during the development of COPD and after CS cessation is largely unknown. In this study, we explored the association between CS-induced changes in diaphragm contractility and mitochondrial alterations in morphology and homeostasis using rat models in response to CS exposure and CS cessation.

\section{Methods}

\subsection{Establishment of the COPD rat model}


Seven-week-old SPF male Sprague-Dawley (SD) rats (Beijing Vital River Laboratory Animal Technology Co., Ltd., Beijing, China) were housed under conditions of 12-hour light/dark cycle, $25-26^{\circ} \mathrm{C}$, and $50-60 \%$ humidity at the animal facility, Beijing Chaoyang Hospital. Rats were given ad libitum access to food and water. All animal procedures approved by the Animal Care and Ethics Committee of Beijing Chaoyang Hospital were performed in accordance with the Guide for the Care and Use of Laboratory Animals of the Beijing Chaoyang Hospital, Capital Medical University. After a 1-week adaptation, the rats were randomly divided into three CS-exposed groups and the respective control groups according to the duration of CS exposure: a 3-month CS group ( $33, n=10)$ with a matched no-smoking control group $(C 3, n=14)$; a 6month CS group (S6, $n=9)$ with a matched control group $(C 6, n=15)$; and a 6-month CS Group followed by 3 months cessation $(S 6 N 3, n=9)$ with a matched control group $(C 9, n=7)$.

The CS group rats were exposed to CS with Baisha Filter cigarettes (tobacco type; tar, 10 mg; nicotine content, $1.0 \mathrm{mg}$; and carbon monoxide, $13 \mathrm{mg}$ ) for $1 \mathrm{~h}$ twice a day. The SIBATA SG-300 oro-nasal cigarette suction system (Shibata Scientific Technology Ltd., Tokyo, Japan) and a CS exposure chamber

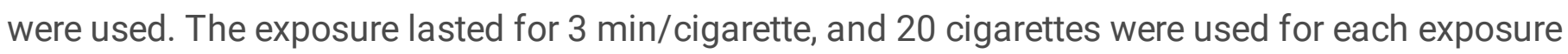
procedure, with a maintained level of total particulate matter of $993.6 \pm 125.7 \mathrm{mg} / \mathrm{m}^{3}$. The control rats were housed under the same conditions without CS exposure. The COPD model was established by evaluating the pulmonary functional changes and lung tissue histological changes for further experiments. The rats were excluded if the rats died prematurely, if the rats did not meet the following experimental schedules and criteria, or if the examples failed to meet quality control standards described below.

\subsection{Pulmonary function test and lung histopathological analyses}

Pulmonary function was assessed usinganAniRes2005 animal lung function analysis system (Beijing Beilanbo Technology). The forced expiratory volume in $0.3 \mathrm{~s}\left(\mathrm{FEV}_{0.3}\right)$, forced vital capacity (FVC), and the ratio of $\mathrm{FEV}_{0.3} / \mathrm{FVC}$ were calculated.

Following the pulmonary functional assay, the rats were sacrificed by exsanguination from the abdominal aorta. The lungs were inflated with $4 \%$ paraformaldehyde solution through the endotracheal intubation at a constant pressure of $25 \mathrm{~cm} \mathrm{H}_{2} \mathrm{O}$ for $10 \mathrm{~min}$. Then, the right lower lobe was removed from rats after ligating the bronchi, fixed in $4 \%$ paraformaldehyde solution for $48 \mathrm{~h}$, and embedded in paraffin. These paraffin-embedded tissues were cut into 5 - $\mu \mathrm{m}$ sections and stained with hematoxylin and eosin (H\&E, Scy Tek Laboratories).

Emphysema, reflecting pulmonary injury, was assessed by measuring the mean linear intercept (MLI) and the mean alveolar number (MAN), as previously described [26]. Ten fields (100x) for the MLI and twenty fields (200x) for the MAN (without the large trachea and blood vessels) were randomly selected and a cross was drawn through the center of each field. The number of alveolar intervals ( $\mathrm{Ni}$ ) lying on the cross, the total length $(\mathrm{L})$ of the cross, the area of the field $(\mathrm{S})$, and the number of alveoli $(\mathrm{Na})$ in each field was determined $(\mathrm{MLI}=\mathrm{L} / \mathrm{Ni}$ and $\mathrm{MAN}=\mathrm{Na} / \mathrm{S})$ using Adobe Photoshop CS5. 
Semi-quantitative histological assessment of H\&E sections was performed blindly using an ordinal scoring system designed to distinguish the degree of lung inflammation [27]. Severity scores ranging from 0 to 4 were used, and severity was assessed by noting the most advanced grade present within the specific sample irrespective of its horizontal extent. The extent was defined as the horizontal distribution of pathology, where a score of $0,1,2$, or 3 meant that none of the lung was involved, $\leq 1 / 3$ involvement, $1 / 2$ involvement, or $\geq 2 / 3$ involvement, respectively. The overall score was defined as a combined assessment of severity and extent (overall score $=$ severity $\times$ extent).

\subsection{Contractile function of the diaphragm in vitro}

Diaphragmatic contractile properties were measured using the RM6240 biological signal acquisition and processing system (Chengdu Instrument Factory, Sichuan, China). The diaphragm was quickly removed and immediately immersed in Kreb's solution containing the following (mmol/l): $118 \mathrm{NaCl}, 4.7 \mathrm{KCl}$, $1.25 \mathrm{CaCl}_{2}, 1.2 \mathrm{MgSO}_{4}, 1.2 \mathrm{KH}_{2} \mathrm{PO}_{4}, 25 \mathrm{NaHCO}_{3}$, and $11 \mathrm{Glucose} ; \mathrm{pH}$ value, 7.3-7.4. A rectangular muscle bundle, $\sim 5 \mathrm{~mm}$ wide, parallel to the long axis of the fibers, was cut in the upper region of the right hemidiaphragm with the central tendon and rib reserved. The diaphragm bundle was placed vertically into the glass bath filled with Kreb's solution at $37^{\circ} \mathrm{C}$ and perfused with a mixture of $95 \% \mathrm{O}_{2}$ and $5 \% \mathrm{CO}_{2}$.

The rib end of the diaphragm was tied to an "L"-shaped metal bar at the bottom of the bath and the other end was connected to the tension transducer. Two silver stimulating electrodes separated by a distance of $1 \mathrm{~cm}$ were inserted into the bundle. After a 30 -min thermo-equilibration period, the bundle was extended to its optimal length (Lo, the length with peak twitch force) and stimulations were delivered through an electronic stimulator. Subsequently, the threshold voltage at Lo was determined, defined as the minimum stimulus intensity that caused the maximum contraction of the diaphragm bundle. The voltage was then increased by $20 \%$ to ensure supramaximal stimulation and this voltage was used during the subsequent experiments.

Twitch characteristics: Two twitches were recorded at Lo to determine the twitch tension (Pt), the maximum tension rise rate $\left(+d T / d t_{\text {max }}\right)$, and the maximum tension fall rate $\left(-d T / d t_{\text {max }}\right)$. The average value for the two twitches was used for further analysis.

Maximal tetanic tension (Po): The bundle was stimulated twice tetanically at $160 \mathrm{~Hz}$ with a $0.2 \mathrm{~ms}$ wave width for $250 \mathrm{~ms}$ to obtain a clear plateau in force generation. The Po was recorded as the plateau tension and the average value for the two stimulations was used.

Force-frequency curve: The bundle was stimulated at the following frequencies: $10,20,40,60,80,100$, and $150 \mathrm{~Hz}$. Each stimulus was separated by a 2-min interval. The curve was plotted with stimulation frequency on the $X$ axis and tension on the $Y$ axis.

Fatigue index (FI):The FI was assessed with a low-frequency protocol in accordance with the modified Burke method [28]. The muscle bundle was fatigued by using 330-ms stimulations repeated at $40 \mathrm{~Hz}$ and 
applied every second for $2 \mathrm{~min}$. The FI was calculated as the force at the end of the 2-min stimulation divided by the maximum force during the initial stimulation.

Standardization of values: The values of Pt and Po were corrected using the cross-sectional area (CSA) of the muscle bundle. The CSA $\left(\mathrm{cm}^{2}\right)$ was calculated by dividing the weight by the specific density $\left(1.056 \mathrm{~g} / \mathrm{cm}^{3}\right)$ and muscle length.

\subsection{Electron microscopy}

Samples of the diaphragm were processed for electron microscopy according to the standard methods at the Electron Microscope Laboratory of Peking University People's Hospital. Briefly, each diaphragm sample was immediately fixed with $3 \%$ glutaraldehyde solution and $1 \%$ osmic acid solution, and then dehydrated with different concentrations of ethanol. The samples were embedded in epoxy resin. Ultrathin sections were obtained along the long axis of muscle fibers and stained using uranyl acetate and lead citrate. The ultrastructure of the diaphragm cells, such as the muscle fiber arrangement, "Z-line," and mitochondrial morphology, was observed and photographed in three copper nets for each sample using an FEI Tecnai Spirit Transmission Electron Microscope at constant calibrated magnifications of $6,000 \times$ and 1,1500x. Ten randomly selected fields from each copper net were analyzed (6,000x). The mitochondrial number density (NA) and mitochondrial volume density (Vv) were established and calculated by Image Scope image analysis software. Ten horizontal and 10 vertical lines were crossdrawn equidistant through the space. $\mathrm{NA}(/ \mu \mathrm{m} 2)=$ mitochondrial number of reference space/whole space area; $\mathrm{Vv}(\%)=$ number of points that hit mitochondria/number of points that hit reference space [29].

\subsection{Quantitative Real-Time polymerase chain reaction (qPCR)}

Total RNA was extracted using TRIzol reagent (Invitrogen, USA). First-strand CDNA synthesis was performed using PrimeScript RT Master Mix (TaKaRa Biotech, Dalian, China). The mRNA levels of genes of interest, such as PTEN induced putative kinase 1 (Pink1), BCL2 interacting protein 3 (Bnip3), mitofusin 1 (Mfn1), mitofusin 2 (Mfn2), optic atrophy 1 (Opa1) and dynamin-related protein 1 (Drp1), were measured by qPCR using SYBR Green Master Mix (TaKaRa Biotech, Dalian, China). The total amount of mRNA was normalized to Actin levels. The primers needed are listed in Table 1.

\subsection{Western Blot}

SDS polyacrylamide gel electrophoresis (SDS-PAGE) was run on 8 or $10 \%$ gradient gels with equal amounts 50ug protein loaded. Relative amounts of Pink1, Bnip3, Mfn1, Opa1 and Tubulin were assessed using the following primary antibodies: (1) Pink1 (product no. ab23707, Abcam, Cambridge区MA); (2) Bnip3 (product no. ab109326, Abcam, Cambridge, MA); (3) Mfn1 (product no.ab104274, Abcam, Cambridge, MA); (4) Opa1 (product no. ab157457, Abcam, Cambridge, MA); (5) Tubulin (product no. ab108342, Abcam, Cambridge, MA). Secondary antibodies (Zhongshan Golden Bridge, Beijing, China) 
were applied at room temperature for 2 hours. Protein bands were visualized using ECL reagent. Image $\mathrm{J}$ software was used for subsequent quantification and statistical analysis.

\subsection{Statistical Analysis}

For normally distributed continuous variables, the data are presented as the mean $\pm S D$, unless stated otherwise. To compare continuous variables, the Kolmogorov-Smirnov test was used to test the normality of the data. Statistical comparisons between two groups were performed via two-tailed Student's t-test for normally distributed continuous variables or the Mann-Whitney U test for nonnormally distributed continuous variables. Statistical comparisons of three or more groups were performed using One-way ANOVA (Least significant difference post hoc test) for normally distributed variables, and one-way Kruskal-Wallis tests (Dunn's post hoc test) for non-normally distributed variables. Statistical significance was set at $p<0.05$. The data were analyzed with SPSS 17.0 software.

\section{Results}

\subsection{CS exposure caused marked structural and functional disruptions, and CS cessation failed to lead to a significant recovery}

Compared to the control rats, the CS-exposed rats had thinner bodies and significantly decreased body weight (Fig. 1A), accompanied with marked disruptions of lung function and structure, along with enhanced infiltration of inflammatory cells within the alveolar cavity and around the bronchi (Fig. 1B-D).

The longer CS exposure led to inflammatory cell infiltration and severe lung structural disruption, as indicated by significant distention of the air spaces and the destruction of the alveolar septa in the lungs of S6 and S6N3 rats (Fig. 1C, D). S6 rats also showed enhanced decrease in MAN (Fig. 1E). Consistently, the histopathology scores further confirmed the progressive adverse structural change in response to CS exposure (Fig. 1E). In line with these structural changes, the lung functional assay showed CS exposureinduced progressive disruption, as indicated by a decrease in the ratio of $\mathrm{FEV}_{0.3} / \mathrm{FVC}$ (Fig. 1B).

Additionally, the CS cessation group exhibited eased lung histological changes induced by CS exposure, including decreased pulmonary inflammation and a limited reversal of emphysema, as indicated by constricted inflammatory infiltration, a thinner alveolar septum, and a significant increase in MAN versus S6 (Fig. 1C-E). However, compared to the age-matched controls (C9), MAN, MLI, and histological score measurements showed significant changes induced by CS, suggesting a limited reversal (Fig. 1E).

\subsection{CS exposure depressed diaphragmatic contractile function, which was eased by 3-month CS cessation}

To evaluate the diaphragmatic contractile function, we measured $\mathrm{Pt} / \mathrm{CSA},+\mathrm{dT} / \mathrm{dt}_{\mathrm{max}}$ $\mathrm{dT} / \mathrm{dt}_{\max }, \mathrm{Po} / \mathrm{CSA}$, and the force-frequency curve to evaluate the strength, and $\mathrm{FI}$ to evaluate the 
endurance. Compared to controls, the diaphragmatic function in $\mathrm{S} 3$ rats exhibited significant decreases in $\mathrm{Pt} / \mathrm{CSA},+\mathrm{dT} / \mathrm{dt}_{\max }$, and $-\mathrm{dT} / \mathrm{dt}_{\mathrm{max}}$, with no changes in Po/CSA and force-frequency curve (Fig. 2A-D, F). Interestingly, the FI of the diaphragms of S3 rats was remarkably increased versus that in the control group, suggesting that the endurance of the diaphragm was enhanced after short-term CS exposure (Fig. $2 \mathrm{E}$ ). These findings suggested that short-term CS exposure promoted an adaptive transition in diaphragmatic function to maintain respiratory function. However, 3-month CS exposure significantly repressed the contractility of the extensor digitorum longus (EDL) muscle (S-Figures 1A-D, F), suggesting that skeletal muscle was more vulnerable to CS exposure. This discrepancy also indicated a distinct regulation between respiratory and skeletal muscle during CS-induced COPD.

Upon the prolongation of CS exposure, the diaphragm function declined, as indicated by the marked decreases in Pt/CSA, dT/max, and Po/CSA in the S6 group (Fig. 2A-D). Furthermore, in these S6 diaphragm, the force-frequency curve is shifted downward and the force increase by stimuli was significantly weaker with the increase in stimulation frequency, along with a markedly depressed force generation under each stimulus frequency ( $p=0.001-0.008)$ (Fig. $2 \mathrm{G}$ ). Additionally, the FI of S6 rats exhibited a trend of increase compared to the $\mathrm{C} 6$ groups, although no statistical difference was achieved (Fig. 2E). These observations suggested progress to maladaptive transition upon long-term CS exposure.

Smoking cessation for three months likely eased the diaphragm contractile function, as evidenced by the fact that S6N3 rat and age-matched controls (C9) exhibited similar diaphragm function (Fig. 2A-E, H). Consistently, S6N3 showed a significant increase in dT/max compared to S6 (Fig. 2C, D), suggesting a beneficial effect of cessation on diaphragm function. The beneficial effects of CS cessation were also observed in EDL muscle (S-Figures 1A-D, H). This interesting observation confirmed the benefits of smoking cessation for smoking patients with COPD.

\subsection{CS exposure caused disruption of mitochondrial morphology in diaphragmatic muscle, while CS cessation eased mitochondrial damage}

We examined diaphragm myofibrils and mitochondrial morphological changes using electron microscopy. Compared to age-paired controls, S3, S6, and S6N3 diaphragms failed to show obvious changes in the morphology of diaphragmatic muscle cells, with clear $Z$ lines and sarcomeres arranged in order (Fig. 3A). However, CS induced marked changes in diaphragmatic mitochondria. S3 rats displayed a slight change in the morphology of diaphragmatic mitochondria, including larger size and more regular distribution, with increased mitochondrial number density, especially in the perinuclear area (Fig. 3A, C). These morphological changes were consistent with the enhanced endurance of diaphragm muscle in S3 rats.

Conversely, the S6 rats exhibited marked morphologic changes in diaphragmatic mitochondria, including obviously fuzzy outlines, vague or missing cristae, and even vacuolar-like degeneration (Fig. 3A). Moreover, the mitochondrial volume density and number density were significantly decreased (Fig. 3B, C). Intriguingly, the morphology and microstructure of the mitochondria in the S6N3 group seemed to be 
recovered, as indicated by clear outlines and cristae and a less vacuolar-like structure. Particularly, the mitochondrial volume density was significantly higher than that of the control (Fig. 3B). The six-month CS exposure caused remarkable disruption of diaphragm mitochondria, which was eased by cessation, likely contribute to the improved diaphragmatic function in S6N3 rats.

\subsection{Long-term CS exposure induced deregulation of mitophagy and fusion/fission, which was alleviated by CS cessation}

To examine whether mitochondrial morphology changes in the diaphragm were associated with alterations in mitophagy and mitochondrial fusion/fission, we measured the expression levels of their key regulators in the diaphragm, including Pink1, Bnip3, Mfn1, Mfn2, Opa1, and Drp1. There was no significant difference in these markers between the $\mathrm{S} 3$ and $\mathrm{C} 3$ groups at both the mRNA and protein levels (Fig. 4A, B), whereas the S6 diaphragms showed significantly decreased expression of Pink1 and Mfn1 (Fig. 4A, C). This finding accorded with the observations of mitochondrial morphology. Of note, both the mRNA and protein levels of Mfn1 were recovered in the CS cessation rat (S6N3) group, whereas Pink1 expression was significantly decreased compared to the C9 controls (Fig. 4A, D). These observed changes in the expression of Pink1 and Mfn1 suggested that the deregulation of mitochondrial quality control was likely involved in CS-induced mitochondrial disruption.

\section{Discussion}

CS is one of the greatest risk factors for the pathogenesis COPD and CS cessation is beneficial in preventing COPD progression, while the effects of cessation on the diaphragm are unknown. We hypothesized that cessation could eased CS-induced diaphragmatic dysfunction and mitochondrial changes would be involved. In the current study, we examined this hypothesis by use CS-exposure rat model. We found that CS exposure caused histological disruption and functional depression in the lungs, and CS cessation failed to result in a significant recovery. CS induced a significant decline in diaphragmatic muscle contractility, which was recovered after 3-month CS cessation. In parallel, the disruption of the mitochondrial morphology in diaphragmatic muscle was significantly alleviated after cessation. These diaphragmatic muscle changes are likely associated with deregulated mitochondrial homeostasis, including mitophagy and mitochondrial fusion/fission.

\section{CS exposure caused progressive irreversible disruption of lung structure: The most commonly} encountered risk factor for COPD is tobacco smoking, and therefore, smoking cessation has the marked capacity to influence the development of COPD $[1,9]$. Smoking cessation has been shown to be most effective intervention to reduce the deterioration of lung function in COPD. However, an even longer duration of smoking cessation cannot fully reverse the lung pathology and inflammation [30-32]. The lesions after smoking cessation may be attributed to the persistent inflammatory changes induced by the elevation of cytokines, such as interleukin-12 and matrix metalloproteinase-12 [31,32]. In this study, CS induced progressive disruption in lung tissue, with enhanced pulmonary inflammation. CS cessation only partially reversed the adverse structural changes including constricted inflammation and clear alveolar 
structure, but failed to alleviate emphysema, as evidenced by the significant difference in MLI and MAN compared to the age-paired controls. This limited amelioration of emphysema reasons that smoking cessation failed to improve significant pulmonary functions.

\section{Mitochondrial alterations were essential for the diaphragmatic transition from adaptive to maladaptive in response to CS exposure: Diaphragmatic dysfunction has been found in patients with COPD, whereas} other studies have indicated that some adaptive changes are beneficial to diaphragm function, such as shorter sarcomere length and increased mitochondrial density [3]. In our CS-exposure rat model, threemonth CS exposure led to an enhanced endurance of the diaphragm, with increased mitochondrial number density, whereas longer-exposure caused an adverse transition to diaphragmatic dysfunction with disrupted mitochondrial morphology. Therefore, the outcome of diaphragm function was dependent on the shift of the balance between harmful factors and adaptive mechanisms, which is associated with the progression of disease, acute exacerbation and treatment intervention $[3,14,33]$.

Previous studies on mice indicated that CS caused mitochondrial dysfunction, accompanied by limb muscle mass loss, and diaphragm muscle atrophy $[3,14,33]$. In our CS-exposure model, although the lungs showed significant structural and functional disruptions compared to the paired controls, no obvious diaphragm atrophy was observed. This could be due to the different animal models, in which the diaphragm muscle in mouse is more susceptible to CS-induced emphysema-like changes than that in the rat. Of note, our models predominantly showed progressive changes in mitochondrial morphology and the expression of quality regulators, Mfn1 and Pink1. These changes might occur earlier than the atrophy of muscle cells. Our findings further emphasize the critical role of mitochondrial regulation in the diaphragm muscle during the development of COPD.

\section{CS cessation alleviated diaphragm dysfunction and mitochondrial morphology damage induced by CS} exposure: A previous study revealed that 2 weeks of smoking cessation could reverse smoking-induced mitochondrial dysfunction and limb muscle mass loss [14]. Consistently, in our rat model, diaphragmatic contractile impairment and mitochondrial destruction induced by 6-month CS exposure were eased after 3 months of smoking cessation. Such an improvement in the diaphragm was considerably more obvious than the recovery in the lung with persistent emphysema, suggesting that the direct effect of CS, as well as depressed respiratory function, contributed to diaphragm dysfunction. It is likely that short-term CSinduced lung function depression is a trigger for adaptive transition of the diaphragm to maintain pulmonary function, whereas, upon long-term CS exposure, the direct effect of CS would be associated with the mitochondrial damage leading to maladaptive transition of the diaphragm. After 3-month smoking cessation, the direct effect reduction of CS contributed to the improvement of diaphragm function and mitochondrial morphology. Our findings using this animal experimental model advanced the knowledge about the beneficial effects of smoking cessation for patients with COPD.

\section{Mitophagy and fusion/fission contribute to diaphragm dysfunction induced by CS exposure: Previous} studies on alveolar epithelial, endothelial, and immune cells have shown a fundamental influence of CSinduced oxidative stress on mitochondrial quality control [21-25]. Additionally, impaired mitochondrial 
structural and functional integrity have been shown to contribute to skeletal muscle atrophy during COPD development, which was associated with increased mitochondrial ROS [16]. Here, consistent with these previous findings, the diaphragm from S6 rats exhibited significantly impaired integrity, suggesting deregulated mitochondrial quality control. Our results provided evidence for the importance of mitochondrial quality control deregulation in respiratory muscle dysfunction during CS-induced COPD.

The mitochondrial quality control is responsible for mitophagy and fusion/fission, which are complicated cellular regulation mechanisms involved in various regulators, including Pink1, PRKN/PARK2, Bnip3, Mfn1, Mfn2, Opa1, and Drp1 [18,21-25]. Here, we found that long-term CS exposure caused significant decreases in Pink1 and Mfn1, suggesting altered mitochondrial quality control.

Mitophagy, as an important mechanism of mitochondrial quality control, selectively eliminates damaged mitochondria via autophagy. Mitophagy is initiated via both Pink1-parkin RBR E3 ubiquitin protein ligase (PRKN)-dependent and -independent pathways. Upon damage, Pink1 recruits and activities PRKN to ubiquitinate mitochondrial outer membrane proteins, leading to the recruitment of autophagy receptors [34]. The deregulation of Pink1-PRKN-mediated mitophagy is involved in mitochondrial ROS production in the lung and skeletal muscle in response to CS exposure [20,21]. Pink1-PRKN-independent mitophagy is mediated directly by a mitochondrial outer membrane protein, Bnip3, which can recruit autophagy receptors after activation. Bnip3 can be activated by hypoxia-inducible factor-1 under hypoxic conditions and protects cells through reducing ROS [35,36]. In our S6 rats, a decrease in Pink1, but not Binp3, was observed in the diaphragm in response to CS exposure, suggesting that CS-induced mitophagy depression is likely associated with a Pink1-PRKN-dependent mechanism. Notably, CS cessation failed to significantly reverse Pink1 expression, suggesting that the CS-induced depression of mitochondrial mitophagy in the diaphragm was persistent. Given the slight recovery observed in diaphragmatic muscle strength after smoking cessation, perhaps a longer cessation time is necessary for recovery.

Previous studies have shown that airway epithelial cells from patients with COPD exhibit defective mitochondrial morphology, likely due to the impaired balance between mitochondrial fission and fusion [25]. Mitochondrial fusion is associated with mitochondrial outer and inner membrane proteins, including Mfn1, Mfn2, and Opa1, while mitochondrial fission is associated with Drp1 and Fis1 [37]. Our results indicated a significantly decreased level of Mfn1 in the $\mathrm{S} 6$ group only, and this decrease was recovered in the CS-cessation group at both the mRNA and protein level consistent with the changes in diaphragmatic muscle strength and mitochondrial morphology. It has been shown that mitochondrial fusion associated with Mfn1 helps to eliminate defective mitochondrial components to maintain normal function [38]. Therefore, decreased Mfn1 expression herein suggested that the imbalance of mitochondrial quality control would be one of the critical causes of CS-induced diaphragmatic dysfunction, and CS-cessation eased such imbalance leading to the recovery of diaphragmatic function.

\section{Conclusion}


Our study revealed dynamic changes in the diaphragm in terms of morphology, microstructure, and contractile properties, in response to different durations of CS exposure and after 3-month smoking cessation. These dysfunctional changes were found together with damaged mitochondrial morphology, which was associated with deregulated mitochondrial homeostasis, including mitophagy and mitochondrial fusion/fission. Moreover, skeletal muscle was more vulnerable to CS exposure, suggesting a distinct regulation between respiratory and skeletal muscle during CS-induced COPD. We also confirmed the inevitability of lung structural changes with persistent emphysema induced by CS exposure, whereas smoking cessation eased CS-induced diaphragmatic dysfunction and mitochondrial deregulation. These diaphragmatic muscle changes are likely associated with deregulated mitochondrial homeostasis, including mitophagy and mitochondrial fusion/fission. These findings advance the understanding of respiratory muscle transitions during the development of COPD, endorsing the importance of mitochondrial homeostasis in the pathogenesis of COPD.

\section{Abbreviations}

COPD: Chronic obstructive pulmonary disease; CS: cigarette smoking; ROS: reactive oxygen species; EDL: extensor digitorum longus; $\mathrm{FEV}_{0.3}$ : forced expiratory volume in 0.3 seconds; FVC: forced vital capacity; H\&E: hematoxylin and eosin; MLI: mean linear intercept; MAN: mean alveolar number; Pt: twitch tension; Po: maximal tetanic tension; $+\mathrm{dT} / \mathrm{dt}_{\text {max }}$ : maximum tension rise rate; $-\mathrm{dT} / \mathrm{dt}_{\text {max }}$ : maximum tension fall rate; Fl: fatigue index; CSA: cross-sectional area. Pink1: PTEN induced putative kinase 1; Bnip3: BCL2 interacting protein 3; Mfn1: mitofusin 1; Mfn2: mitofusin 2; Opa1: optic atrophy 1; Drp1, dynamin-related protein 1.

\section{Declarations}

\section{Authors' contributions}

ZY, SH, MY, JJ and PB conceived and designed the experiments. ZY, SH, MY and JJ analyzed and interpreted the results of the experiments. ZY, SH, SX and HY performed the experiments. ZY and JJ wrote the initial draft of the manuscript. All authors have read and approved the manuscript.

\section{Ethics approval and consent to participate}

All animal procedures approved by the Animal Care and Ethics Committee of Beijing Chaoyang Hospital were performed in accordance with the Guide for the Care and Use of Laboratory Animals of the Beijing Chaoyang Hospital, Capital Medical University.

\section{Consent for publication}

Not applicable.

\section{Availability of data and materials}


The datasets used and/or analysed during the current study are available from the corresponding author on reasonable request.

\section{Competing interests}

The authors declare that they have no competing interests.

\section{Funding}

Not applicable.

\section{Acknowledgments}

Not applicable.

\section{References}

1. Global Initiative for Chronic Obstructive Lung Disease (GOLD) Global Strategy for the Diagnosis,

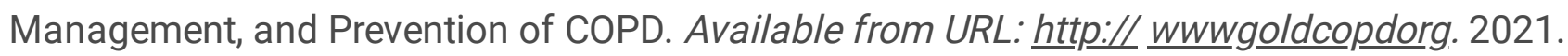

2. Vilaró J, Ramirez-Sarmiento A, Martínez-Llorens JM, et al. Global muscle dysfunction as a risk factor of readmission to hospital due to COPD exacerbations. Respiratory medicine. 2010;104(12):18961902.

3. Barreiro E, Gea J. Respiratory and Limb Muscle Dysfunction in COPD. COPD. 2015;12(4):413-426.

4. Doucet $M$, Debigare $R$, Joanisse $D R$, et al. Adaptation of the diaphragm and the vastus lateralis in mild-to-moderate COPD. Eur Respir J. 2004;24(6):971-979.

5. Ottenheijm CA, Heunks LM, Dekhuijzen RP. Diaphragm adaptations in patients with COPD. Respir Res. 2008;9:12.

6. Sheng $\mathrm{H}$, Zhang $\mathrm{Y}$, Shi $\mathrm{X}$, et al. Functional, Ultrastructural, and Transcriptomic Changes in Rat Diaphragms with Different Durations of Cigarette Smoke Exposure. Int J Chron Obstruct Pulmon Dis. 2020;15:3135-3145.

7. Doucet $M$, Dube A, Joanisse DR, et al. Atrophy and hypertrophy signalling of the quadriceps and diaphragm in COPD. Thorax. 2010;65(11):963-970.

8. Nucci RAB, de Souza RR, Suemoto CK, et al. Cigarette Smoking Impairs the Diaphragm Muscle Structure of Patients without Respiratory Pathologies: An Autopsy Study. Cell Physiol Biochem. 2019;53:648-655.

9. Fragoso CA. Epidemiology of Chronic Obstructive Pulmonary Disease (COPD) in Aging Populations. COPD. 2016;13(2):125-129.

10. Raherison C, Girodet PO. Epidemiology of COPD. Eur Respir Rev. 2009;18(114):213-221.

11. Barreiro E, Peinado VI, Galdiz JB, et al. Cigarette smoke-induced oxidative stress: A role in chronic obstructive pulmonary disease skeletal muscle dysfunction. Am J Respir Crit Care Med. 
2010;182(4):477-488.

12. Maltais F, Decramer M, Casaburi R, et al. An official American Thoracic Society/European Respiratory Society statement: update on limb muscle dysfunction in chronic obstructive pulmonary disease. $A m$ J Respir Crit Care Med. 2014;189(9):e15-62.

13. Nogueira L, Trisko BM, Lima-Rosa FL, et al. Cigarette smoke directly impairs skeletal muscle function through capillary regression and altered myofibre calcium kinetics in mice. The Journal of physiology. 2018;596(14):2901-2916.

14. Ajime TT, Serré J, Wüst RCl, et al. Two Weeks of Smoking Cessation Reverse Cigarette SmokeInduced Skeletal Muscle Atrophy and Mitochondrial Dysfunction in Mice. Nicotine \& tobacco research : official journal of the Society for Research on Nicotine and Tobacco. 2021;23(1):143-151.

15. Zhang B, Li P, Li J, Liu X, Wu W. Effect of Oxidative Stress on Diaphragm Dysfunction and Exercise Intervention in Chronic Obstructive Pulmonary Disease. Frontiers in Physiology. 2021;12(805).

16. Gouspillou G, Godin R, Piquereau J, et al. Protective role of Parkin in skeletal muscle contractile and mitochondrial function. The Journal of physiology. 2018;596(13):2565-2579.

17. Peker N, Donipadi V, Sharma M, McFarlane C, Kambadur R. Loss of Parkin impairs mitochondrial function and leads to muscle atrophy. Am J Physiol Cell Physiol. 2018;315(2):C164-C185.

18. Racanelli AC, Kikkers SA, Choi AMK, Cloonan SM. Autophagy and inflammation in chronic respiratory disease. Autophagy. 2018;14(2):221-232.

19. Taivassalo T, Hussain SN. Contribution of the Mitochondria to Locomotor Muscle Dysfunction in Patients With COPD. Chest. 2016;149(5):1302-1312.

20. Mao J, Li Y, Feng S, et al. Bufei Jianpi Formula Improves Mitochondrial Function and Suppresses Mitophagy in Skeletal Muscle via the Adenosine Monophosphate-Activated Protein Kinase Pathway in Chronic Obstructive Pulmonary Disease. Frontiers in pharmacology. 2020;11:587176.

21. Mizumura K, Cloonan SM, Nakahira K, et al. Mitophagy-dependent necroptosis contributes to the pathogenesis of COPD. The Journal of clinical investigation. 2014;124(9):3987-4003.

22. Ito $S$, Araya J, Kurita Y, et al. PARK2-mediated mitophagy is involved in regulation of HBEC senescence in COPD pathogenesis. Autophagy. 2015;11(3):547-559.

23. Ahmad T, Sundar IK, Lerner CA, et al. Impaired mitophagy leads to cigarette smoke stress-induced cellular senescence: implications for chronic obstructive pulmonary disease. FASEB journal : official publication of the Federation of American Societies for Experimental Biology. 2015;29(7):2912-2929.

24. Araya J, Tsubouchi K, Sato N, et al. PRKN-regulated mitophagy and cellular senescence during COPD pathogenesis. Autophagy. 2019;15(3):510-526.

25. Jiang $Y$, Wang $X$, Hu D. Mitochondrial alterations during oxidative stress in chronic obstructive pulmonary disease. Int J Chron Obstruct Pulmon Dis. 2017;12:1153-1162.

26. Yu N, Sun YT, Su XM, He M, Dai B, Kang J. Treatment with eucalyptol mitigates cigarette smokeinduced lung injury through suppressing ICAM-1 gene expression. Bioscience reports. 2018;38(4). 
27. Silva RM, Anderson DS, Franzi LM, et al. Pulmonary effects of silver nanoparticle size, coating, and dose over time upon intratracheal instillation. Toxicological sciences : an official journal of the Society of Toxicology. 2015;144(1):151-162.

28. Burke RE, Levine DN, Zajac FE, 3rd. Mammalian motor units: physiological-histochemical correlation in three types in cat gastrocnemius. Science (New York, NY). 1971;174(4010):709-712.

29. Hoppeler H, Mathieu O, Krauer R, Claassen H, Armstrong RB, Weibel ER. Design of the mammalian respiratory system. VI Distribution of mitochondria and capillaries in various muscles. Respir Physiol. 1981;44(1):87-111.

30. Lapperre TS, Postma DS, Gosman MM, et al. Relation between duration of smoking cessation and bronchial inflammation in COPD. Thorax. 2006;61(2):115-121.

31. Braber S, Henricks PA, Nijkamp FP, Kraneveld AD, Folkerts G. Inflammatory changes in the airways of mice caused by cigarette smoke exposure are only partially reversed after smoking cessation. Respir Res. 2010;11:99.

32. Yan H, Zhao L, Wu X, et al. Inflammation and pathological damage to the lungs of mice are only partially reversed following smoking cessation on subacute exposure to cigarette smoke. Mol Med Rep. 2015;11(6):4246-4254.

33. Bowen TS, Aakerøy L, Eisenkolb S, et al. Exercise Training Reverses Extrapulmonary Impairments in Smoke-exposed Mice. Medicine and science in sports and exercise. 2017;49(5):879-887.

34. Geisler S, Holmström KM, Skujat D, et al. PINK1/Parkin-mediated mitophagy is dependent on VDAC1 and p62/SQSTM1. Nature cell biology. 2010;12(2):119-131.

35. Zhang H, Bosch-Marce M, Shimoda LA, et al. Mitochondrial autophagy is an HIF-1-dependent adaptive metabolic response to hypoxia. The Journal of biological chemistry. 2008;283(16):1089210903.

36. Bellot G, Garcia-Medina R, Gounon P, et al. Hypoxia-induced autophagy is mediated through hypoxiainducible factor induction of BNIP3 and BNIP3L via their BH3 domains. Molecular and cellular biology. 2009;29(10):2570-2581.

37. Wai T, Langer T. Mitochondrial Dynamics and Metabolic Regulation. Trends Endocrinol Metab. 2016;27(2):105-117.

38. Gomes LC, Di Benedetto G, Scorrano L. During autophagy mitochondria elongate, are spared from degradation and sustain cell viability. Nature cell biology. 2011;13(5):589-598.

\section{Tables}

Table 1 Primers used for qPCR analysis for genes 


\begin{tabular}{cll} 
Gene & Forward primer & Reverse primer \\
\hline Actin & GCAGGAGTACGATGAGTCCG & ACGCAGCTCAGTAACAGTCC \\
\hline Pink1 & GCTTCCGCCTGGAGGATTAT & GGGCATGGTGGCTTCATACA \\
\hline Bnip3 & ATTGGTCAAGTCGGCCAGAA & GAGAGTAGCTGTGCGCTTCG \\
\hline Mfn1 & TGACTTGGACTACTCGTGCG & GACTTGGTGGCTGCAGTTTG \\
\hline Mfn2 & GGCACAGAGGAGACCTCAAG & AAGTGCTTGAGAGGGGAAGC \\
\hline Opa1 & CAGTTCAGAAGACCTCGCCA & CAGGTGTACCCGCAGTGAAG \\
\hline Drp1 & TGGAAAGAGCTCAGTGCTGG & CAACTCCATTTTCTTCTCCTGTTGT
\end{tabular}

\section{Figures}


Figure 1

A

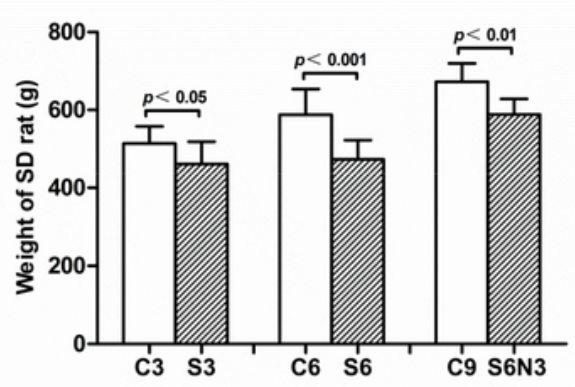

B

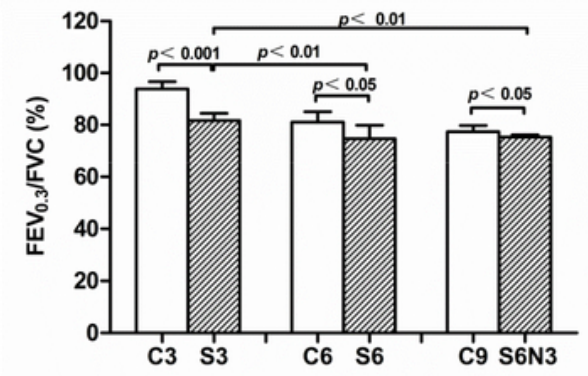

C

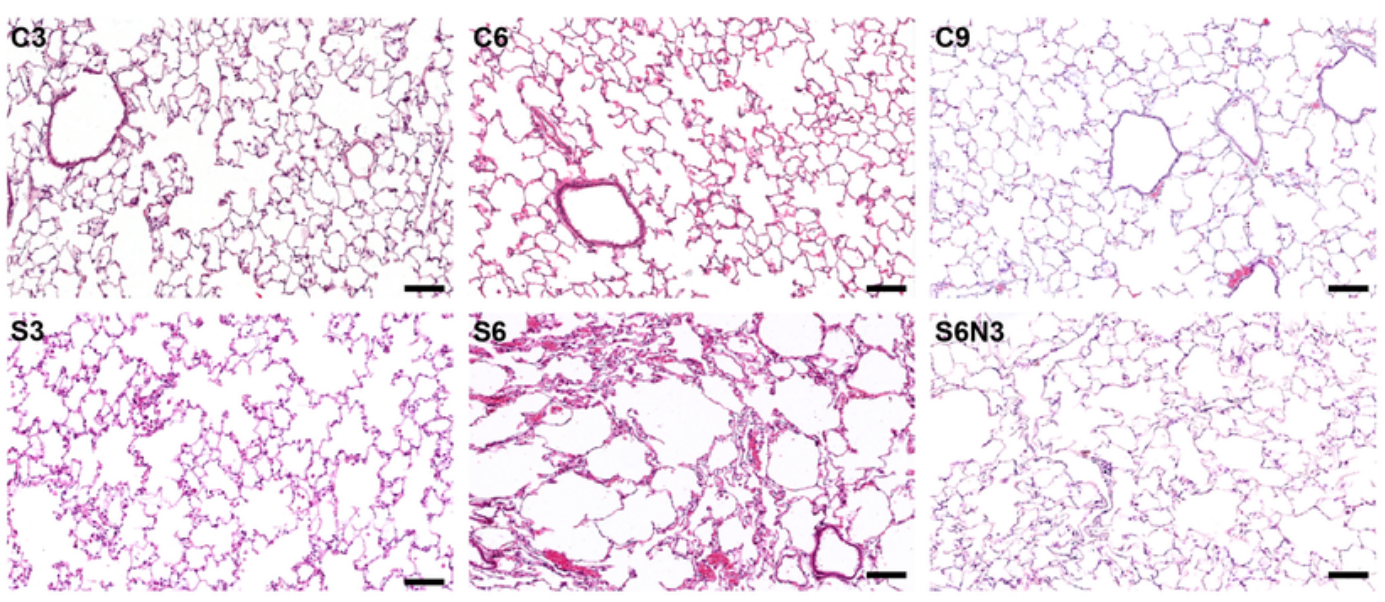

D

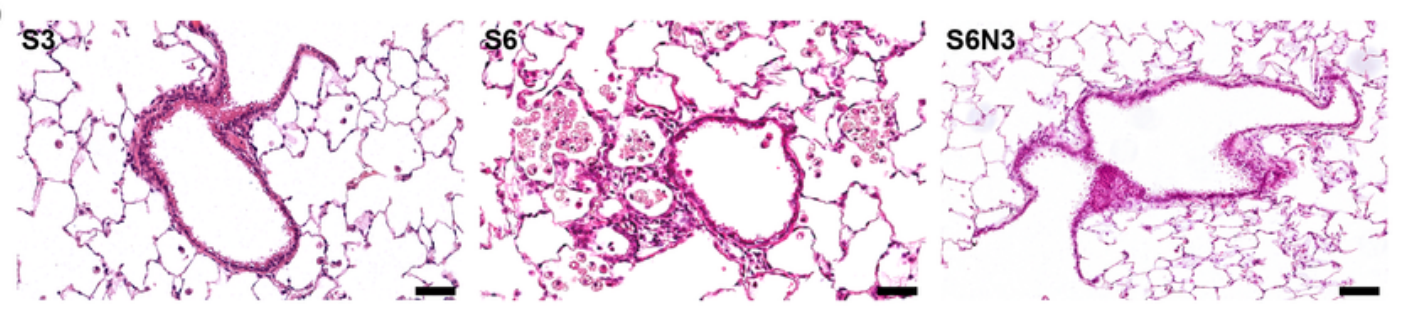

E
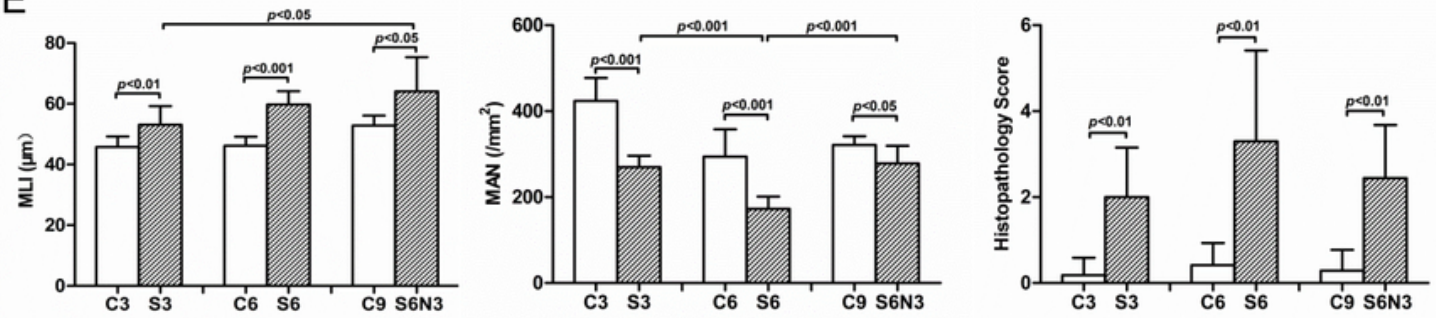

\section{Figure 1}

CS exposure caused marked structural and functional disruptions and CS cessation failed to cause a significant recovery

A. CS-exposed rats showed decreased body weight versus non-exposed controls. B. CS exposure caused a significant decrease in $\mathrm{FEV}_{0.3} / \mathrm{FVC}$, and the S6 and S6N3 groups exhibited severe decreases compared 
to the S3 group; there was no significant difference in $\mathrm{FEV}_{0.3} / \mathrm{FVC}$ between the $\mathrm{S} 6$ and S6N3 groups. C and D. Representative H\&E-stained images of lung tissue. Significant distention of the alveolar cavity with destruction of the alveolar septa and reduced alveolar units were observed in the lungs of S3, S6, and S6N3 rats compared to the control rats $(100 \times)$ (C). The S6 lungs showed more severe changes than S3 and S6N3 lungs (C). Increased inflammatory cell infiltration within the alveolar cavity and around the bronchi in the lungs from S3, S6, and S6N3 rats compared to the control rats (200x) (D). E. Compared to the control rats, in all CS groups (S3, S6, and S6N3), the MLI and the histopathology scores significantly increased, and MAN decreased. The S6 rats showed enhanced decrease in MAN versus the S3 rats, while the CS cessation group (S6N3) exhibited a significant increase in MAN versus S6, rather than MLI and the histopathology scores, indicating limited reversal of emphysema. Data are presented as the mean $\pm S D, n$ $=6-15$ per group. Scale bar: $100 \mu \mathrm{m}$ for $\mathbf{C} ; 50 \mu \mathrm{m}$ for $\mathbf{D}$. 
Figure 2

A

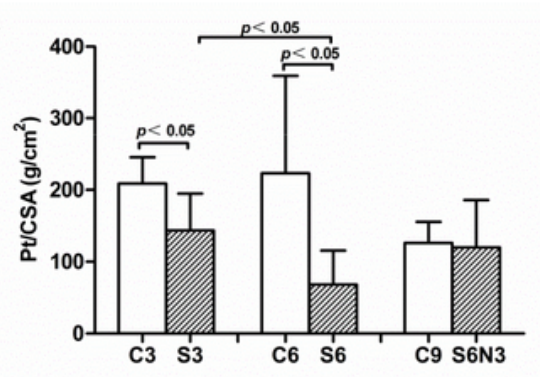

C

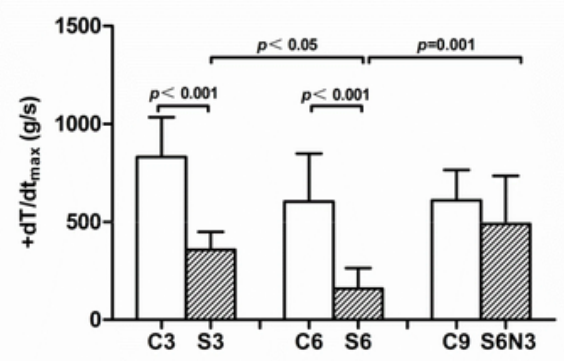

E

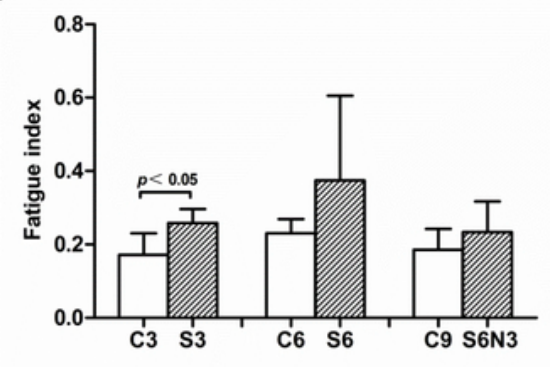

G

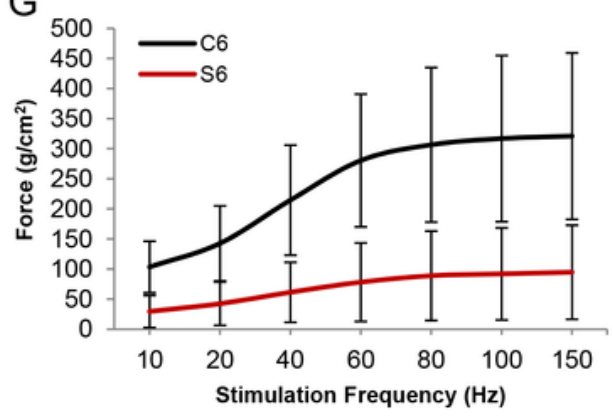

B

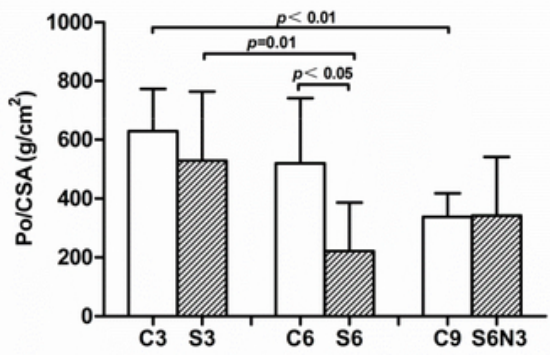

D

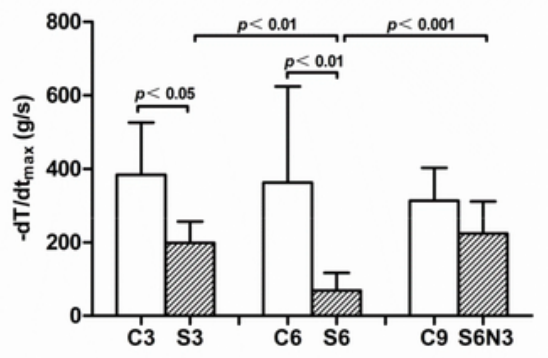

$\mathrm{F}$

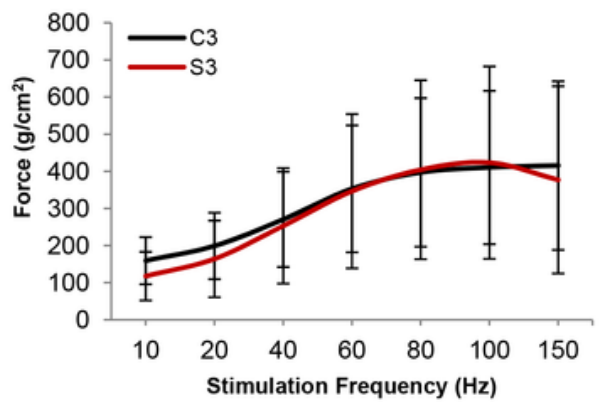

$\mathrm{H}$

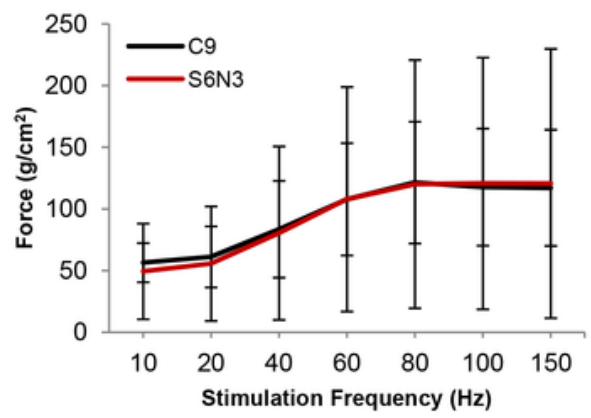

Figure 2

CS exposure depressed diaphragmatic contractile function, and CS cessation eased CS-induced contractile decline

A. Compared to controls, the Pt/CSA was significantly decreased in S3 and S6 rats, but not in S6N3 rats. Upon the prolongation of CS exposure, the Pt/CSA of the S6 rats was worse than that of the S3 rats. B. 
The Po/CSA exhibited a significant decrease in S6 rats, but not in S3 and S6N3 rats. C-D. The $+d T / d t_{\text {max }}$ and $-\mathrm{dT} / \mathrm{dt}_{\max }$ were significantly decreased in $\mathrm{S} 3$ and $\mathrm{S} 6$ rats, but not in S6N3 rats. Upon the prolongation of CS exposure, $\mathrm{S} 6$ rats showed worse declines of $+\mathrm{dT} / \mathrm{dt} \mathrm{t}_{\max }$ and $-\mathrm{dT} / \mathrm{dt} \mathrm{t}_{\max }$ versus the $\mathrm{S} 3$ rats. After smoking cessation for 3 months (S6N3), the $+d T / d t_{\max }$ and $-d T / d t_{\max }$ were significantly improved compared to those in the S6 rats. E. The FI of the diaphragm from S3 rats was remarkably increased compared to that in the control group, suggesting that the endurance of the diaphragm was enhanced after short-term CS exposure. F-H. Force-frequency curve of diaphragm for the CS groups and the controls. There was no significant difference in force-frequency curve between S3 and C3 groups (F). In the S6 diaphragm, the force-frequency curve is shifted downward and the force increase was significantly weaker with the increase of stimulation frequency, along with a markedly depressed force generation under each stimulus frequency $(p=0.001-0.008)(\mathbf{G})$. Smoking cessation for 3 months likely eased the diaphragm contractile function. The S6N3 rat and age-matched controls (C9) exhibited similar in forcefrequency curve $(H)$. Data are presented as the mean $\pm S D, n=6-8$ per group. 
Figure 3

A
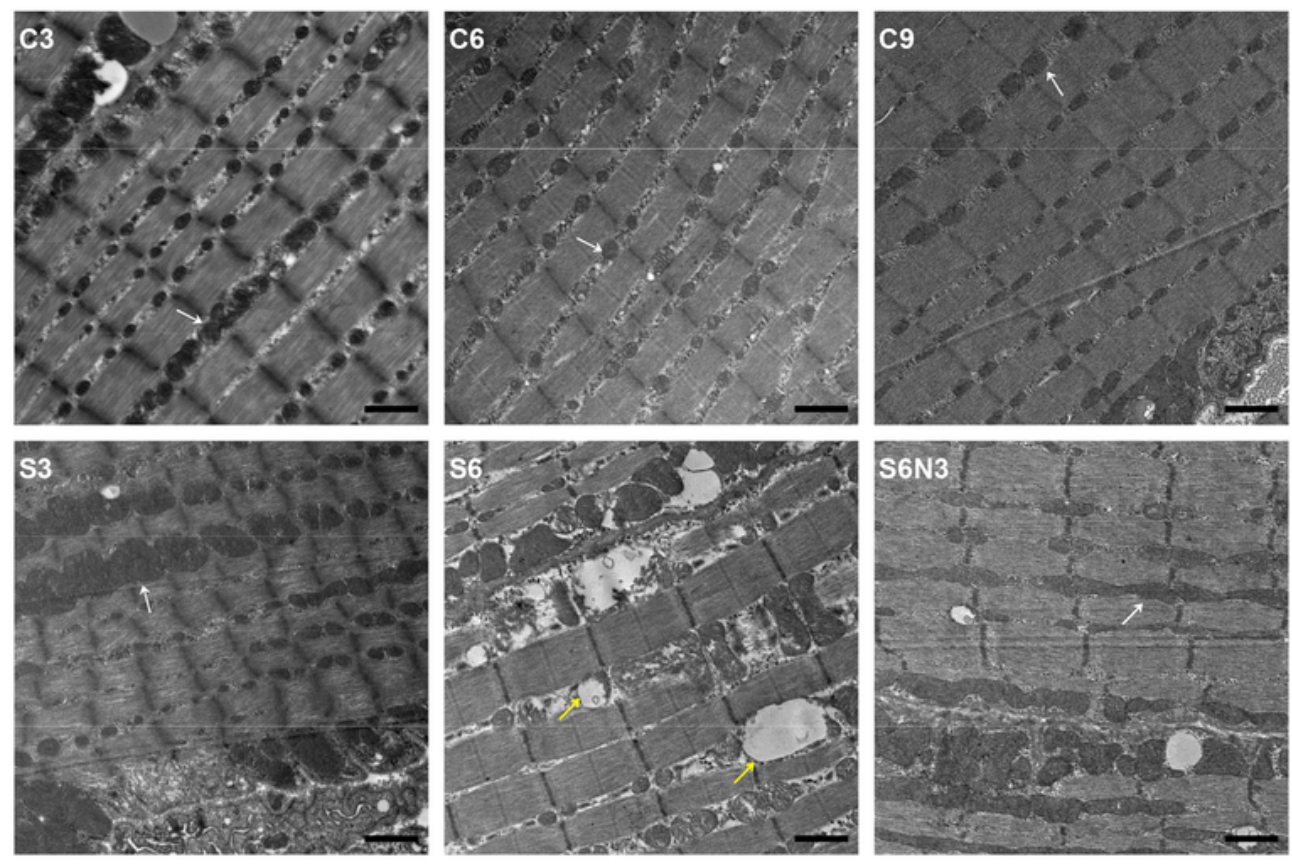

B

C
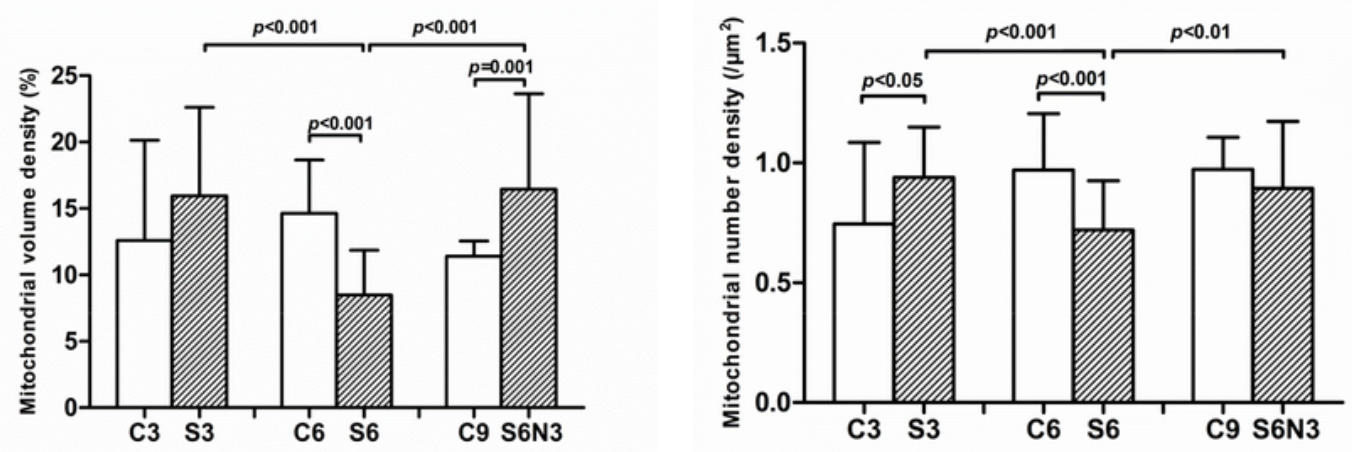

Figure 3

CS exposure caused disruption of mitochondrial morphology in diaphragmatic muscle, and CS-cessation ameliorated mitochondrial damages

A. As shown by the ultrastructure of diaphragm (11500x), S3, S6 and S6N3 diaphragms failed to show significant changes in the morphology of diaphragmatic muscle cells, with clear Z lines and sarcomeres 
arranged in order. S3 diaphragm displayed a slight change in morphology of diaphragmatic mitochondria (white arrow) with increased mitochondrial number, while the $\mathbf{S 6}$ diaphragm exhibited marked morphologic changes in diaphragmatic mitochondria, including obviously fuzzy outlines, vague or missing cristae, and even vacuolar-like degeneration (yellow arrow). The morphology and microstructure of the mitochondria in the S6N3 group seemed to be recovered as indicated by clear outlines and cristae and a less vacuolar-like structure. B-C. The mitochondrial volume density (B) and number density (C) were measured. The S3 rats displayed an increased mitochondrial number density. Upon the prolongation of CS exposure, the $\mathrm{S} 6$ rats exhibited significant decreases in both mitochondrial volume density and number density. After smoking cessation for 3 months (S6N3), both mitochondrial volume density and number density showed improved compared to the $\mathrm{S} 6$ rats, especially the mitochondrial volume density significantly increased versus the control (C9). Data presented as mean $\pm S D, n=6$ per group. Scale bar: 1 $\mu \mathrm{m}$ for $\mathbf{A}$. 
Figure 4
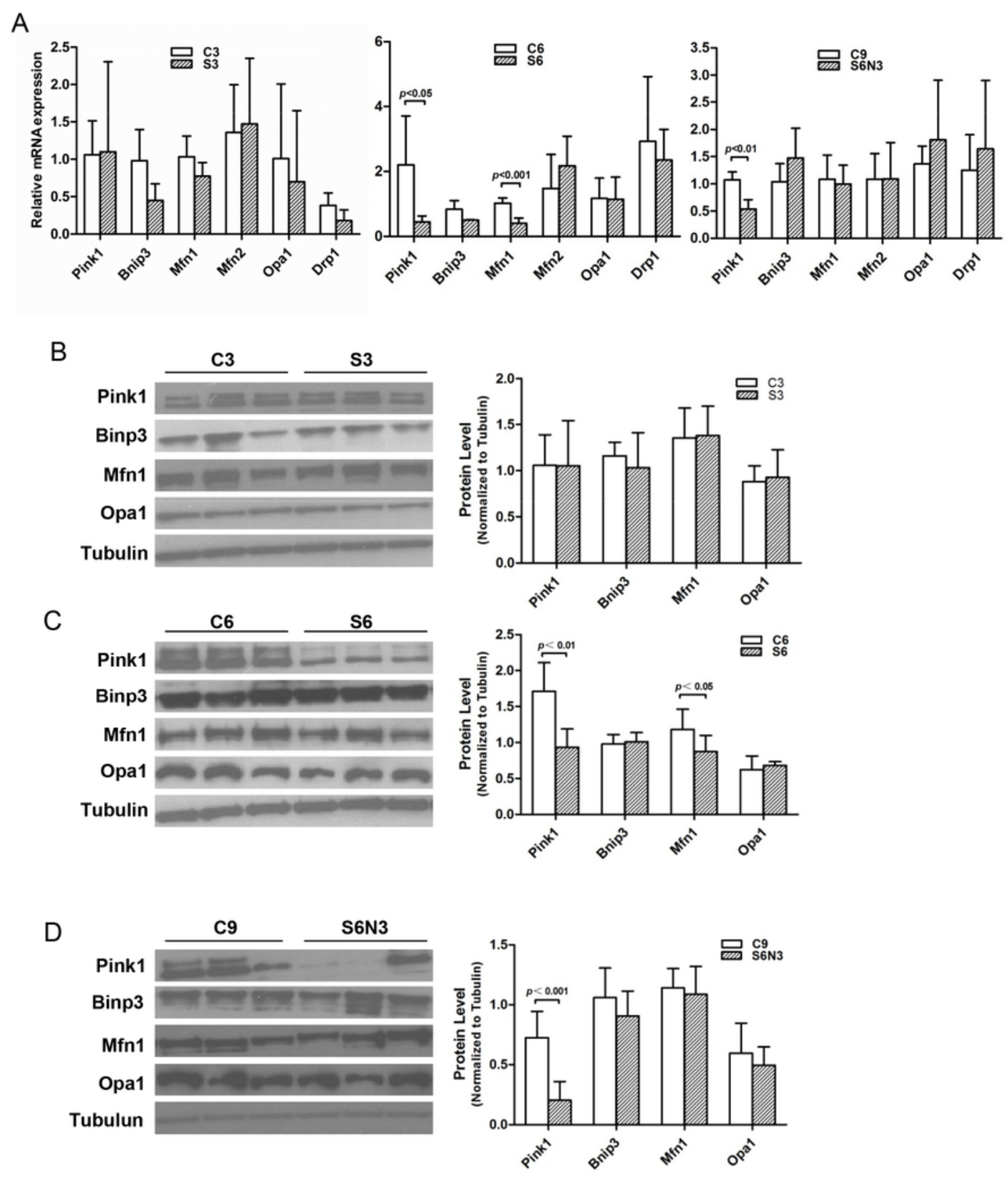

\section{Figure 4}

Comparison of expression levels of key regulators related to mitophagy and fusion/fission in the rat diaphragm

There was no significant difference in these markers between the $\mathrm{S} 3$ and $\mathrm{C} 3$ groups at both the mRNA and protein levels (A, B). The S6 diaphragms showed significantly decreased expression of Pink1 and 
Mfn1 at both mRNA and protein levels (A, C). In the CS cessation (S6N3) group, both the mRNA and protein levels of Mfn1 were recovered, whereas Pink1 expression was significantly decreased versus $\mathrm{C} 9$ controls (A, D).

\section{Supplementary Files}

This is a list of supplementary files associated with this preprint. Click to download.

- AuthorChecklistE10only.pdf

- sfigureandlegend.pdf 